3 Bechdolf A, Pukrop R, Kohn D, Tschinkel S, Veith V, Schultze-Lutter F, et al. Subjective quality of life in subjects at risk for a first episode of psychosis: a comparison with first episode schizophrenia patients and healthy controls Schizophr Res 2005; 79: 137-43.

4 Francey SM, Jackson HJ, Phillips LJ, Wood SJ, Yung AR, McGorry PD. Sustained attention in young people at high risk of psychosis does not predict transition to psychosis. Schizophr Res 2005; 79: 127-36.

5 Koike $S$, Takano $Y$, Iwashiro $N$, Satomura $Y$, Suga $M$, Nagai $T$, et al. A multimodal approach to investigate biomarkers for psychosis in a clinical setting: the integrative neuroimaging studies in schizophrenia targeting for early intervention and prevention (IN-STEP) project. Schizophr Res 2013; 143 116-24.

Matteo Rocchetti, Department of Psychosis Studies, Institute of Psychiatry Psychology and Neuroscience (IOPPN), King's College London, UK, and Department of Brain and Behavioral Sciences, University of Pavia, Via Bassi 21, 27100, Pavia, Italy Email: matteo.rocchetti01@universitadipavia.it; Alberto Sardella, Alessia Avila, Department of Psychosis Studies, IOPPN, King's College London, UK; Martina Brandizzi, Department of Psychosis Studies, IOPPN, King's College London, UK, and Brandizzi, Department of Psychosis Studies, lOPPN, King's College London, UK,
Neurosciences, Mental Health and Sensory Functions (NESMOS) Department, Sapienza University of Rome, Rome, Italy; Edgardo Caverzasi, Pierluigi Politi, Department of Brain and Behavioral Sciences, University of Pavia, Pavia, Italy; Stephan Ruhrmann, Department of Psychiatry and Psychotherapy, University of Cologne, Cologne, Germany; Philip McGuire, Paolo Fusar-Poli, Department of Psychosis Studies, IOPPN, King's College London, and OASIS Prodromal Team, South London and the Maudsley NHS Foundation Trust, London, UK

doi: 10.1192/bjp.208.2.197a

\section{Childhood environment and intergenerational transmission of depression}

Plant and colleagues, in a very interesting and elegant study, ${ }^{1}$ found that maternal depression during pregnancy was associated with: offspring depression in adulthood (odds ratio (OR) 3.4), maternal depression during offspring's childhood $(\mathrm{OR}=4.8)$, and offspring exposure to child maltreatment $(\mathrm{OR}=2.4)$. However, as the authors said, 'when childhood factors (i.e. child maltreatment, maternal depression 1-16 years) were entered at the second steps, prenatal maternal depression no longer predicted significantly offspring depression'. In our view, these findings are suggestive that the key causal factor is not maternal depression during pregnancy, but maternal depression during offspring childhood and child maltreatment (probably the former promoting the later). Since maternal depression during pregnancy probably does not directly cause child maltreatment or later maternal depression, these two should not be viewed as mediators or mechanisms of the association found between maternal depression during pregnancy with offspring depression in adulthood. Since after adding the childhood factors there is no statistical correlation of maternal depression during pregnancy with offspring depression in adulthood anymore, this seems suggestive that maternal depression during pregnancy is more probably a marker of mothers with higher risk of developing depression during offspring childhood and of offering/allowing maladaptive parental behaviour. This is in line with previous studies showing that environmental factors, especially maladaptive parental behaviour, were total or partial mediators of the association between parental and offspring depressive symptoms. ${ }^{2-4}$ Despite maternal depression during pregnancy being a marker of an at-risk mother-child dyad, the actual causal factors seem to be the factors happening during childhood: maternal depression and parental behaviour. So, preventive measures should focus on screening mothers with depression (during pregnancy, but especially during offspring childhood), providing treatment and support for adequate parental behaviour.

However, the authors' conclusions go in the opposite direction. In the paper's discussion, it is stated 'we did not find that exposure to maternal depression after birth contributes to this association (maternal depression during pregnancy with offspring depression in adulthood). This suggests that exposure to maternal depression specifically during pregnancy represents a unique setting for the intergenerational transmission of risk for depression'. However, the results section states 'offspring exposure to maternal depression during childhood (1-16 years) was associated significantly with offspring adulthood depression $(\mathrm{OR}=4.2)$ '. They see their study 'in line with the theoretical premise of fetal programming', related to elevated levels of maternal glucocorticoids at the intrauterine environment. Finally, for preventive measures, they emphasise screening and treating expectant mothers with depression, supporting the use of antidepressants during pregnancy. These are valuable measures, but not supported by this study results.

In summary, in our perspective, this extremely well-done study supports the view that childhood factors (parental behaviour and maternal depression) have key causal implications on intergenerational transmission of depression. Preventive measures should focus mainly on childhood, providing treatment and support for adequate parental behaviour.

1 Plant DT, Pariante CM, Sharp D, Pawlby S. Maternal depression during pregnancy and offspring depression in adulthood: role of child maltreatment. Br J Psychiatry 2015; 207: 213-20.

2 Johnson JG, Cohen $\mathrm{P}$, Kasen S, Smailes E, Brook JS. Association of maladaptive parental behavior with psychiatric disorder among parents and their offspring. Arch Gen Psychiatry 2001; 58: 453-60.

3 Tully EC, Iacono WG, McGue M. An adoption study of parental depression as an environmental liability for adolescent depression and childhood disruptive disorders. Am J Psychiatry 2008; 165: 1148-54.

4 Elgar FJ, Mills RS, McGrath PJ, Waschbusch DA, Brownridge DA. Maternal and paternal depressive symptoms and child maladjustment: the mediating role of parental behavior. J Abnorm Child Psychol 2007; 35: 943-55.

Alexander Moreira-Almeida, Associate Professor of Psychiatry, University Hospita Alexander Moreira-Almeida, Associate Professor of Psychiatry, University Hosp
and School of Medicine, Federal University of Juiz de Fora (UFJF), BraziL. Email: alex.ma@ufjf.edu.br; Mauro Junqueira de Souza, University Hospital, UFJF, Brazil

doi: 10.1192/bjp.208.2.198

Authors' reply: We thank Moreira-Almeida \& Junqueira de Souza for their interesting correspondence on our paper. Indeed, we regard childhood environmental factors as highly important to the intergenerational pathways for the transmission of depression. Nevertheless, maternal depression during pregnancy in itself has been identified as a significant risk factor for offspring depression, not only in our sample, but also in other samples of varying demographics and size. ${ }^{1,2}$

There are plausible and documented mechanisms linking a mother's depression in pregnancy with her child's increased vulnerability to experiencing maltreatment; namely, changes to the mother-child attachment relationship, maladaptive caregiving behaviours, interparental conflict and increased offspring reactive temperament. ${ }^{3}$ Such mechanisms likely operate by compromising levels of care and protection afforded by a mother, as well as directly affecting stress resiliency in her developing child, thereby increasing her child's vulnerability to being exposed to, and experiencing, episodes of maltreatment. Depression is a disorder with a recurrent course, ${ }^{4}$ thereby meaning the likelihood of depression after birth is elevated following an episode during pregnancy. Additionally, the temporal precedence of antenatal depression to childhood maltreatment and further maternal depression after birth adds to the logic as to why antenatal depression should be considered as a primary risk factor in the intergenerational transmission of depression, and the aforementioned childhood adversities as mediators to this trajectory.

As Moreira-Almeida \& Junqueira de Souza correctly highlight, in our multiple hierarchical regression models, maternal depression during pregnancy was not found to predict offspring adulthood 